\title{
A Case of Herpetic Keratitis after Subconjunctival Triamcinolone Acetonide Injection
}

\author{
Hidenori Inoue Takashi Suzuki Takeshi Joko Tomoyuki Inoue \\ Yuichi Ohashi \\ Department of Ophthalmology, Ehime University, Graduate School of Medicine, \\ Toon, Japan
}

\section{Key Words}

Herpetic keratitis - Subconjunctival triamcinolone acetonide injection - Anterior uveitis .

Polymerase chain reaction · Hypotony

\begin{abstract}
Purpose: We report a case of herpetic epithelial keratitis that developed after subconjunctival triamcinolone acetonide injection (STI). Methods: A 65-year-old female with anterior uveitis and hypotony in her right eye was given a STI $(2 \mathrm{mg} / 0.5 \mathrm{ml})$. After the injection, she developed redness and an ocular discharge. A clinical examination was performed and realtime polymerase chain reaction (PCR) was used to amplify the viral DNA in a corneal scraping. Results: Slit-lamp biomicroscopy revealed a severe purulent discharge, conjunctival injection, and a geographic corneal ulcer in the right eye. Herpes simplex virus 1 DNA was identified in the corneal scraping using real-time PCR. Herpetic keratitis was diagnosed and topical acyclovir ointment as well as systemic valacyclovir were started. The inflammation subsided with this medication. Conclusion: We encountered a case of herpetic epithelial keratitis after a STI.

(c) 2014 S. Karger AG, Basel
\end{abstract}

\section{Introduction}

Hypotony is a severe complication of the uveitis and can result in marked vision loss [1]. To treat hypotony, the inflammation of the ciliary body must be reduced and aqueous humor production restored. Steroids and immunosuppressive drugs are used to treat uveitis and scleritis. Subconjunctival triamcinolone acetonide injection (STI) is used to treat anterior scleritis and uveitis [2-5]. The reported adverse events of STI are elevated intraocular

Takashi Suzuki, PhD, MD

Department of Ophthalmology

Ehime University, Graduate School of Medicine

Shitsukawa, Toon, Ehime 791-0295 (Japan)

E-Mail t-suzuki@m.ehime-u.ac.jp 
Inoue et al.: A Case of Herpetic Keratitis after Subconjunctival Triamcinolone Acetonide Injection

pressure (IOP), cataract formation or progression, subconjunctival hemorrhage, scleral necrosis, scleral perforation, infectious scleritis, and endophthalmitis $[4,6,7]$. We report a case of herpetic epithelial keratitis after STI for hypotony caused by scleritis and anterior uveitis.

\section{Case Report}

A 65-year-old female who had diabetes mellitus visited a private clinic and presented with injection and visual disturbance in her right eye. Anterior uveitis was diagnosed, and she was referred to our hospital. Slit-lamp biomicroscopy revealed scleral injection, inflammatory cells in the anterior chamber as well as iris synechiae. The IOP in the right eye was $4 \mathrm{~mm} \mathrm{Hg}$ and B-scan ultrasonography showed choroidal detachment. The patient did not have a history of herpetic eye disease. We diagnosed noninfectious anterior uveitis and began steroid treatment with a $0.1 \%$ dexamethasone ophthalmic solution every hour, $1 \%$ atropine eye drops every night, and oral prednisolone $25 \mathrm{mg} /$ day. One month after treatment, the inflammation in the anterior chamber and choroidal detachment subsided while the IOP increased to $15 \mathrm{~mm} \mathrm{Hg}$. In addition, her blood sugar increased due to the adverse effects of oral prednisolone. Therefore, the systemic steroid was tapered and we continued topical steroids. The scleral injection, hypotony, and choroidal detachment reappeared after tapering the systemic steroids. We performed STI to reduce the anterior segment inflammation. She complained of redness, foreign body sensation, and ocular discharge 1 week after STI. Slit-lamp biomicroscopy showed conjunctival injection, discharge, blepharitis with vesicles of the lid margin, and geographic corneal and conjunctival epithelial defects (fig. 1, fig. 2). The patient was suspected of having herpetic keratitis. The topical steroid was stopped and she was treated with topical acyclovir (3\% eye ointment 5 times/day) and oral valacyclovir $(3,000 \mathrm{mg} /$ day). Real time polymerase chain reaction (PCR) of a corneal scraping was performed in order to detect a potential human herpes virus, including herpes simplex virus (HSV) types 1 and 2, varicella-zoster virus, Epstein-Barr virus, cytomegalovirus, and human herpes viruses 6, 7, and 8 . The real-time PCR results were positive only for HSV DNA $\left(3 \times 10^{9} \mathrm{copies} /\right.$ sample). Since the conjunctival injection and epithelial defect improved with the therapy, the valacyclovir was tapered within 1 month. The scleritis and the inflammation in the anterior chamber as well as her herpetic keratitis were controlled with topical acyclovir. However, the hypotony persisted and the cataract progressed markedly. Therefore, a lensectomy and vitrectomy with silicone oil tamponade was performed and the IOP increased to $10 \mathrm{~mm}$ Hg. Thereafter, the patient was administered topical acyclovir and steroids, without any uveitis recurrence.

\section{Discussion}

We reported a patient with herpetic keratitis after STI. The reactivation of latent HSV-1 is associated with several stressors, including drugs and temperature. Local immunosuppression such as corticosteroid use can exacerbate herpetic keratitis [8]. Herpetic keratitis has been reported after using steroids in eye drops, the subconjunctival injection of dexamethasone, and intravitreal triamcinolone injection $[8,9]$.

To our knowledge, however, a reported case of herpetic keratitis after STI has not yet been reported. STI is used for resistant scleritis because triamcinolone is a long-acting synthetic glucocorticoid. Sohn et al. [4] demonstrated the efficacy of STI for nonnecrotizing, 
Inoue et al.: A Case of Herpetic Keratitis after Subconjunctival Triamcinolone Acetonide Injection

noninfectious anterior scleritis. They treated 68 eyes of 53 patients with STI, and 66 eyes (97\%) improved after 1 injection. Although 14 eyes (2.9\%) developed an increased IOP, none of the cases developed scleral necrosis or melt [4]. A few cases of infectious scleritis or endophthalmitis after STI have been reported $[6,7]$. Although our patient did not show keratitis while receiving systemic steroids and steroid eye drops, STI can reactivate HSV and trigger severe herpetic keratitis. The STI likely reactivated the HSV rather than the use of systemic steroids and steroid eye drops. However, it is possible that systemic steroids and steroid eye drops reactivate HSV-1 immediately before the occurrence of keratitis and STI finally induces keratitis. The clinical findings included geographic epithelial defects caused by HSV, and real time PCR demonstrated high HSV-DNA copy numbers. Diabetes is also a risk factor for HSV reactivation [10] and can be related to the herpetic keratitis that developed in our case.

Although the etiology of the anterior uveitis in our case is unknown, HSV could be associated with the pathogenesis because antiviral treatment along with steroids reduced the inflammation in the anterior chamber. Furthermore, herpetic dermatitis along with herpetic keratitis appeared after STI. A primary HSV-1 infection in the ocular site, including eye and eyelid, could be considered, and anterior uveitis could be caused by HSV-1. Therefore, we should consider herpetic keratitis when STI is used to treat unilateral anterior uveitis or scleritis. Although anterior uveitis caused by HSV-1 accompanies IOP elevation, hypotony is rare. HSV-1 could induce a massive inflammation in the ciliary body and reduce aqueous humor production.

In conclusion, STI can associate with the reactivation of HSV epithelial keratitis. We should be cautious when treating patients with unilateral anterior uveitis or scleritis with a history of diabetes mellitus and herpetic eye disease.

\section{Disclosure Statement}

The authors have no commercial or financial interests associated with the article.

\section{References}

1 Daniel E, Pistilli M, Pujari SS, et al: Risk of hypotony in noninfectious uveitis. Ophthalmology 2012;119:2377-2385.

-2 Tu EY, Culbertson WW, Pflugfelder SC, et al: Therapy of non-necrotizing anterior scleritis with subconjunctival corticosteroid injection. Ophthalmology 1995;102:718-724.

-3 Zamir E, Read RW, Smith RE, et al: A prospective evaluation of subconjunctival injection of triamcinolone acetonide for resistant anterior scleritis. Ophthalmology 2002;109:798-805, discussion 805-797.

-4 Sohn EH, Wang R, Read R, et al: Long-term, multicenter evaluation of subconjunctival injection of triamcinolone for non-necrotizing, noninfectious anterior scleritis. Ophthalmology 2011;118:1932-1937.

5 Athanasiadis Y, Tsatsos M, Sharma A, et al: Subconjunctival triamcinolone acetonide in the management of ocular inflammatory disease. J Ocul Pharmacol Ther 2013;29:516-522.

-6 Singh R, Gupta V, Chakarborty A, et al: Fungal endophthalmitis complicating subconjunctival injection of triamcinolone acetonide in anterior scleritis. Ann Ophthalmol (Skokie) 2006;38:253-256.

7 Gharaee H, Khalife M, Poor SS, et al: Infectious scleritis after subtenon triamcinolone acetonide injection. Ocul Immunol Inflamm 2011;19:284-285.

Ostler HB: Glucocorticoid therapy in ocular herpes simplex. I. Limitations. Surv Ophthalmol 1978;23:35-43.

9 Shtein RM, Stahl RM, Saxe SJ, et al: Herpes simplex keratitis after intravitreal triamcinolone acetonide. Cornea 2007;26:641-642.

10 Kaiserman I, Kaiserman N, Nakar S, et al: Herpetic eye disease in diabetic patients. Ophthalmology 2005;112:2184-2188. 
Case Reports in

Ophthalmology
Case Rep Ophthalmol 2014;5:277-280

DOI: $10.1159 / 000367582$

noue et al.: A Case of Herpetic Keratitis after Subconjunctival Triamcinolone

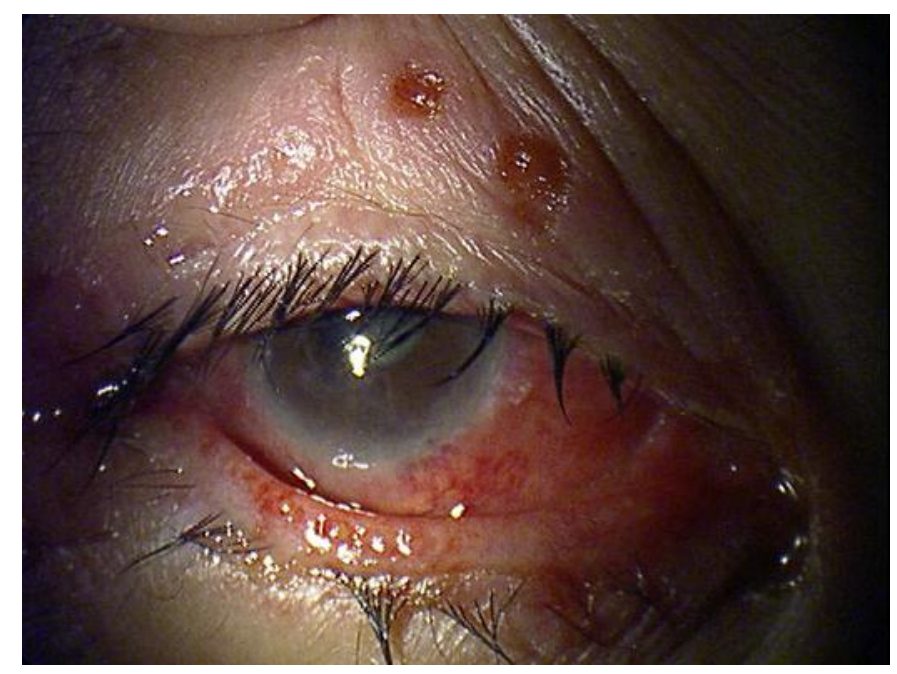

Fig. 1. A photograph showing blepharitis with vesicles of the lid margin and conjunctival injection.

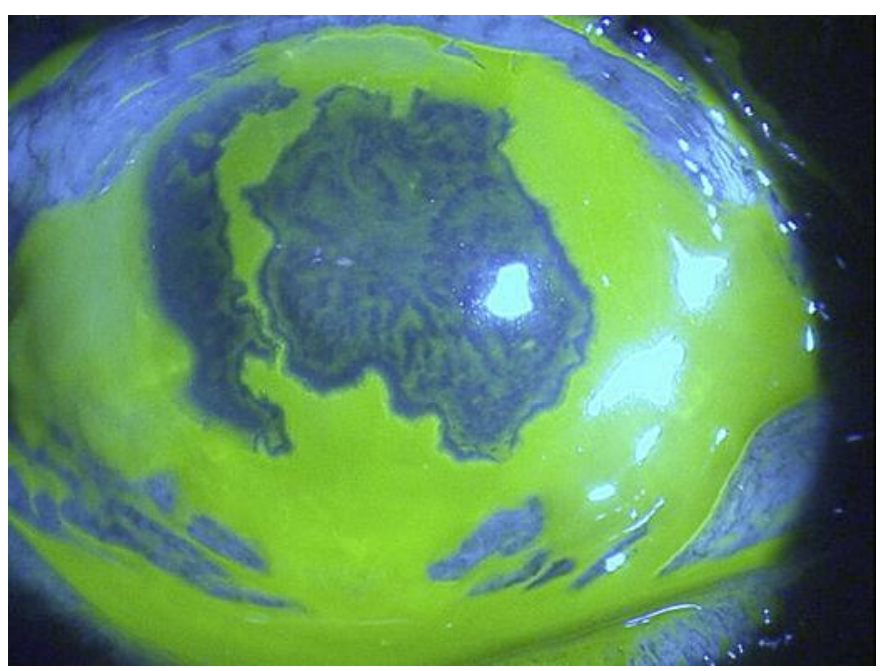

Fig. 2. A photograph showing geographic corneal and conjunctival epithelial defects. 